Original article

\title{
Hematological profile and biochemical markers of COVID-19 non-survivors: A retrospective analysis
}

\author{
Mukesh Bairwa $^{\text {a }}$, Rajesh Kumar ${ }^{\text {b,*, }}$ Kalpana Beniwal ${ }^{\mathrm{b}}$, Deepjyoti Kalita ${ }^{\mathrm{c}}$, Yogesh Bahurupi ${ }^{\mathrm{d}}$ \\ ${ }^{\text {a }}$ Department of Internal Medicine, All India Institute of Medical Sciences (AIIMS), Rishikesh, Uttarakhand, India \\ ${ }^{\mathrm{b}}$ Department of Nursing, All India Institute of Medical Sciences (AIIMS), Rishikesh, Uttarakhand, India \\ ${ }^{\mathrm{c}}$ Department of Microbiology, All India Institute of Medical Sciences (AIIMS), Rishikesh, Uttarakhand, India \\ d Department of Community \& Family Medicine, All India Institute of Medical Sciences (AIIMS), Rishikesh, Uttarakhand, India
}

\section{A R T I C L E I N F O}

\section{Keywords:}

COVID-19

Critical illness

Biomarkers

L-lactate dehydrogenase

C-Reactive protein

\begin{abstract}
A B S T R A C T
Background: Coronavirus disease is primarily transmitted through the respiratory route and bodily contact. The fatality in COVID-19 cases was alarming in the initial days. This study analyzes hematological and biochemical markers of COVID-19 non-survivors.

Material and methods: In this single-center study, records of 249 patients hospitalized with COVID-19 were studied for hematological profile and biochemical markers. Records of patients with laboratory-confirmed COVID-19 disease hospitalized between April 14, 2020, to August 15, 2020, were included in the analysis. Results: Significantly, the disease mortality was associated with increased procalcitonin $(P<0.05)$, C-reactive protein $(P<0.05)$, aspartate transaminase $(P<0.05)$, serum potassium $(P<0.05)$, neutrophils count $(P<0.05)$, white blood cell count $(P<0.05)$, prothrombin time $(P<0.05)$ and activated prothrombin time $(P<0.05)$ in patients reported abnormal x-ray findings. Further, patients with abnormal radiological findings significantly showed a reduced level of lymphocyte counts $(P<0.05)$, oxygen saturation $(P<0.05)$, and partial oxygen pressure $(P<0.05)$. Reduced level of aspartate aminotransferase $(P<0.05)$, alanine aminotransferase $(P<0.05)$ and lactate dehydrogenase $(P<0.05)$ reported significant association with mortality among patients with COVID-19.

Conclusions: The clinicians may consider the hematological and biochemical parameters in the patients with COVID-19 in future decision-making. These indicators might support clinical decisions to identify high fatality cases and poor diagnosis in the initial admission phase. In COVID-19 patients, we recommend close monitoring on procalcitonin, C-reactive protein, neutrophils count, and white blood cell count as a clinical indicator for potential progression to critical illness.
\end{abstract}

\section{Introduction}

Coronavirus disease 2019 was first reported in Wuhan city, Hubei, China, in the last week of December 2019. ${ }^{1}$ The disease reported in a cluster of atypical pneumonia cases and primarily transmitted through respiratory and body contact. ${ }^{2}$ Patients with coronavirus disease demonstrated a series of clinical symptoms, including raised body temperature, cough, headache, nausea, vomiting, anorexia, diarrhea, dyspnea, multiple organ dysfunctions. ${ }^{3}$ A large proportion of infected patients reported mild symptoms of the disease and recover. ${ }^{4}$ Some patients progressively develop serious complications, including sepsis, acute respiratory failure, metabolic acidosis, heart failure, kidney injury, hypoxic encephalopathy, and eventually die of the illness. ${ }^{3}$ A recent report reported a few new symptoms, including anosmia and ageusia. ${ }^{5}$ Considering high transmission and infectivity patterns, World Health Organisation announces it as an emergency of public health concern on March 31, 2020. ${ }^{6}$ In the initial phase of the disease outbreak, the mortality ranges from 2 to $5 \%$, much higher in the elderly. ${ }^{3}$ The mortality in coronavirus cases admitted in Wuhan city reached $7 \%$ in the outbreak's initial days. ${ }^{7}$

Early identification of severe illness risk factors can help clinicians facilitate appropriate remedial measures and help control mortality. ${ }^{8}$ Earlier existing literature on laboratory-confirmed coronavirus cases reported changes in the patients' biochemical parameters, including

\footnotetext{
* Corresponding author. Departement of Nursing, All India Institute of Medical Sciences (AIIMS), Rishikesh, Uttarakhand, 249203, India.

E-mail address: rajeshrak61@gmail.com (R. Kumar).
} 
lymphocyte count, neutrophil count, and D-dimer status. ${ }^{4,9}$ Another study reported changes in inflammatory markers in patients with COVID-19, including C-reactive protein (CRP), erythrocyte sedimentation rate (ESR), and Interleukin-6. ${ }^{3}$ Likewise, another work reported lymphocytopenia, high blood sugar, gamma-glutamyl transferase (GGT), high lactate dehydrogenase (LDH) in more COVID-19 patients. ${ }^{10}$ Further, laboratory findings of 77 COVID-19 deaths and 852 COVID-19 patients also demonstrated an increase in urea, cardiac troponin, creatine kinase, D-dimer, C-reactive protein (CRP), lactate dehydrogenase (LDH), IL-6, and lower level of lactic acid levels and lymphocytes. ${ }^{11,12}$ Analysis of 143 cases of COVID-19 revealed a higher level of C-reactive protein, D-dimer, lactate dehydrogenase, serum amyloid, and lower level of albumin are directly associated with developing a critical illness. ${ }^{9}$

This study performed a profile evaluation of 252 patients with COVID-19 hospitalized at a tertiary care teaching hospital, Northern India. The study retrospectively reviewed the Case files of patients with COVID-19 and compared the clinical and biochemical characteristics between survivors and non-survivors' cases, which may guide clinicians to identify severe cases initially and facilitate appropriate supportive management.

\section{Material \& method}

\subsection{Ethical consideration}

The study was approved by the Institutional Ethics Committees (AIIMS/IEC/20/442). The medical record department was informed before reviewing the record. The need for written consent was deferred considering the research design.

\subsection{Data collection}

All the records of patients with laboratory-confirmed COVID-19 disease $^{13}$ hospitalized in one of the apex health care teaching center, North India, were extracted for a detailed review. Data available included haematological profile, biochemical markers, coagulation profile and arterial blood gas measures in the record of patients hospitalized with COVID-19. Findings on socio-demographic characteristics, initial symptoms and physical examination are under considered for publication in other journal.

\subsection{RNA extraction of virus}

As per ICMR/Govt. of India guidelines, Nasopharyngeal and Nasal swab were collected in Viral Transport Medium and sent to our State Level Viral Research Diagnostic Laboratory (VRDL) situated within the Institute. ${ }^{14}$ In the lab, viral RNA was extracted using a magnetic bead-based automated system Kingfisher Flex system 96 well RNA extractor (ThermoFisher scientific) and MagMax Viral RNA extraction kit (Thermo Fisher Scientific). ${ }^{15}$ About $200 \mu \mathrm{L}$ of sample and $5 \mu \mathrm{L}$ bacteriophage MS2 mixture was processed with $60 \mu \mathrm{L}$ Viral RNA yield. Extracted RNA was processed for real-time Reverse transcriptase-polymerase chain reaction (rt-PCR) using a commercial multiplex rt-PCR kit for SARS CoV-2 detection (Taqpath Covid-19 combo kit by Thermo Fisher Scientific) and Quantstudio-7 real-time Thermocycler (Thermo Fisher Scientific). Briefly, $25 \mu \mathrm{L}$ of reaction volume $(6.25 \mu \mathrm{L}$ of the master mix, $1.25 \mu \mathrm{L}$ of assay mix, i.e., primers and probes for N, S, ORF1b and MS2 targets, $7.5 \mu \mathrm{L}$ of nuclease-free water, and $10 \mu \mathrm{L}$ of RNA extracts) underwent real-time PCR at recommended thermal condition, i.e., Uracil-N-Glycosylase incubation at 250C for $2 \mathrm{~min}$ ( 1 cycle), Reverse transcription at 530C for $10 \mathrm{~min}$ (1 cycle), Activation at 950C for $2 \mathrm{~min}$ ( 1 cycle), Denaturation at 950C and Annealing/Extension at 600C (last 2 stages sequentially for 40 cycles). At the end of PCR cycles, amplification curves (fluorescence) were checked at different preset dye channels, e.g., FAM (ORF1b), VIC (N gene), ABY (S gene), and JUN (MS2). The cut off Cycle Threshold (Ct-value) was 35 (as per kit manufacturer's recommendation), and $\mathrm{Ct}$ values below this were considered positive for SARS-CoV-2 infection.

\subsection{Statistical analysis}

Data are transformed into a Microsoft Excel spreadsheet and analyzed using Statistical Package for Social Sciences (SPSS) Version 23. Descriptive statistics; Mean and standard deviations were used for the comparison of continuous variables ensuring normal distribution. Median and interquartile range (IQR) was used to present the findings. Independent sample -t-test was applied to compare survivors' biochemical and hematological findings to non-survivors in COVID-19 patients. The level of significance was set at $p<0.05$ (two-sided).

\subsection{Case definitions}

The management guidelines issued by Govt. of India, Ministry of Health \& Family Welfare (MoHFW), Directorate General of Health Services (DGHS) for treating SARS-COV-2 patients are used to define a Case. As per the guidelines, a case with respiratory rate $<30$ breaths/ min with severe respiratory problems and $\mathrm{SpO} 2<90 \%$ at room air along with fever and suspected respiratory infection stated a critical case for COVID-19. Further, bilateral opacities unexplained by effusion, lobar and lung collapse, or nodules on chest imaging (x-ray, CT scan, or lung ultrasound) were considered a case with acute respiratory distress syndrome (ARDS). ${ }^{16}$ This study undertakes imaging study findings (X-ray), biochemical, and hematological changes among patients hospitalized with COVID-19. In the present study, survivors included the patients hospitalized with COVID-19 and discharged after undergoing supportive management at the intensive care unit. Mortality refers to death due to COVID-19 infection during hospitalization. Earlier scientific work also stated admission of a patient in an ICU, use of a ventilator, and death as an indicator of critical illness in patients with COVID-19. ${ }^{17,18}$

\section{Results}

\subsection{Hematological profile}

The study is a part of a primary study under consideration for publication in the journal of infection and public health (See Supplementary File in Appendix). The mean age of 249 COVID-19 patients was 45.15 (16.35) range, 7-85 years. 58 patients died with a median age of 55 years old, which was significantly higher than the survivors' group $(P<$ $0.05)$. Blood cell analysis showed that neutrophil counts were significantly higher in the death group than survivors $(P<0.05)$. Besides, the lymphocyte count remains significantly higher in the survivors' cohort $(P<0.05)$. However, there is no significant difference in white blood cells and platelet count between the survivors and death cohort (all $P>$ 0.05). In terms of hemoglobin estimation, the survivors' cohort significantly reported a higher hemoglobin level than the death cohort $(P<$ 0.05). Besides, there was no significant difference in the D-dimer level between the groups ( $\mathrm{P}>0.05)$. (Table $1 \& 2$ ).

Further, the hematological profile is compared based on $\mathrm{x}$-ray findings in patients with COVID-19. Findings showed that white blood cells, neutrophils count, and platelet count was significantly higher among patients with abnormal X-ray findings than their counterparts (all $P<$ 0.05). However, the lymphocyte count was significantly higher in the cohort with normal $\mathrm{x}$-ray findings $(P<0.05)$. Besides, there was no significant difference in hemoglobin in both the cohorts $(P<0.05)$. The duration of partial thromboplastin and activated thromboplastin time was significantly higher among the cohort reported abnormal x-ray findings (all $P<0.05$ ) (Table 3 ). 
Table 1

Clinical Characteristics of patients with COVID-19 ( $\mathrm{N}=249)$.

\begin{tabular}{|c|c|c|c|}
\hline Clinical characteristics & $\begin{array}{l}\text { Normal } \\
\text { range }\end{array}$ & Mean \pm SD & Median (IQR)/n \\
\hline $\begin{array}{l}\text { White blood cell count, } \times \\
10^{9} / \mathrm{L}\end{array}$ & $3.5-4.5$ & $7.7 \pm 4.3$ & $7.4(4.9-9.3) / 247$ \\
\hline $\begin{array}{l}\text { Neutrophils count, } \times 10^{9} / \\
\text { L }\end{array}$ & $1.8-6.3$ & $\begin{array}{l}69.09 \pm \\
15.54\end{array}$ & $6.9(5.9-8.3) / 247$ \\
\hline $\begin{array}{l}\text { Lymphocytes count, } \times \\
10^{9} / \mathrm{L}\end{array}$ & $1.1-3.2$ & $1.60 \pm 1.37$ & $2.1(1.2-3.1) / 247$ \\
\hline Platelet, $\times 10^{9} / \mathrm{L}$ & $125-350$ & $\begin{array}{l}191.29 \pm \\
92.24\end{array}$ & $181(137-231) / 247$ \\
\hline $\mathrm{Hb}, \mathrm{g} / \mathrm{L}$ & $11.5-15.0$ & $12.40 \pm 2.34$ & $\begin{array}{l}12.72(11.14-4.65) / \\
247\end{array}$ \\
\hline PT, s & $9-14$ & $14.46 \pm 6.40$ & $13(12-14.28) / 240$ \\
\hline aPTT, s & $20-40$ & $\begin{array}{l}34.34 \pm \\
14.01\end{array}$ & $\begin{array}{l}31.2(27.9-35.05) / \\
93\end{array}$ \\
\hline Serum sodium, mmol/L & $135-145$ & $\begin{array}{l}138.49 \pm \\
5.57\end{array}$ & $139(136-142) / 246$ \\
\hline $\begin{array}{l}\text { Serum potassium, mmol/ } \\
\mathrm{L}\end{array}$ & $3.5-4.5$ & $4.25 \pm 0.74$ & $4.2(3.8-4.2) / 246$ \\
\hline Serum chloride, mmol/L & 95-105 & $\begin{array}{l}95.77 \pm \\
10.35\end{array}$ & $97(94-99) / 244$ \\
\hline AST, U/L & $8-40$ & $\begin{array}{l}58.26 \pm \\
78.92\end{array}$ & $\begin{array}{l}35.9(24.1-59.8) / \\
244\end{array}$ \\
\hline ALT, U/L & $5-35$ & $\begin{array}{l}203.53 \pm \\
141.59\end{array}$ & $36(24.8-62.3) / 246$ \\
\hline LDH, U/L & $109-245$ & $\begin{array}{l}729.20 \pm \\
621.44\end{array}$ & $\begin{array}{l}513.3(425.7-817) / \\
46\end{array}$ \\
\hline Direct bilirubin, mmol/L & $0.1-0.3$ & $0.65 \pm 2.10$ & $0.3(0.22-0.42) / 246$ \\
\hline $\begin{array}{l}\text { Indirect bilirubin, } \mathrm{mmol} / \\
\mathrm{L}\end{array}$ & $0.2-0.8$ & $0.54 \pm 1.59$ & $\begin{array}{l}0.26(0.18-0.39) / \\
245\end{array}$ \\
\hline TBIL, mmol/L & $3.4-20.5$ & $1.19 \pm 3.63$ & $0.56(0.4-0.8) / 246$ \\
\hline $\mathrm{CRP}, \mathrm{mg} / \mathrm{L}$ & $0-5$ & $\begin{array}{l}56.73 \pm \\
89.87\end{array}$ & $\begin{array}{l}10.15(2.46-79.25) / \\
145\end{array}$ \\
\hline D-dimer, $\mathrm{ng} / \mathrm{mL}$ & $<250$ & $\begin{array}{l}723.69 \pm \\
702.43\end{array}$ & $623(333-908) / 55$ \\
\hline CPK-MB & $0-25$ & $\begin{array}{l}38.72 \pm \\
39.35\end{array}$ & $\begin{array}{l}27.2(18.55-39.05) / \\
85\end{array}$ \\
\hline Procal, ng/mL & $<.004$ & $8.50 \pm 16.97$ & $0.19(.04-5.4) / 55$ \\
\hline $\mathrm{PaO} 2, \mathrm{mmHg}$ & 75-100 & $\begin{array}{l}88.25 \pm \\
14.74\end{array}$ & $98(84-92) / 249$ \\
\hline $\mathrm{SpO} 2, \%$ & $95-100$ & $94.40 \pm 6.62$ & $96(94-98) / 248$ \\
\hline
\end{tabular}

LDL low-density lipoprotein; HDL high-density lipoprotein; TBIL total bilirubin; ALT alanine aminotransferase; AST aspartate transaminase; CK-MB creatinine kinase muscle and brain; APTT activated partial thromboplastin time; PT prothrombin time; CRP C -reactive protein; Proca procalcitonin; Hb hemoglobin.

\subsection{Blood biochemical profile, inflammatory markers, and coagulation profile}

Analysis of the biochemical profile showed that the death cohort significantly has higher serum potassium $(P<0.05)$ than the survivors' group. However, there was no significant difference observed in serum sodium and chloride level in both the groups (all $P>0.05$ ). Besides, the level of aspartate transaminase (AST)) and alanine transaminase (ALT) reported to be significantly (all $P<0.05$ ) higher among the death cohort indicates more severe liver injury in the group. However, there was no significant changes observed in the status of direct bilirubin, indirect bilirubin, and total bilirubin between the two groups (all $P>0.05$ ). Creactive protein and procalcitonin level reported significantly higher among the death cohort in contrast to survivors (all $P<0.05$ ). However, there was no significant difference $(P>0.05)$ observed for CPK-MB level in both groups. Further, the level of prothrombin activated prothrombin time and lactate dehydrogenase (LDH) reported significantly higher (all $P<0.05$ ) in the death cohort. In contrast, the level of partial oxygen pressure $(\mathrm{PaO} 2)$ and oxygen saturation $(\mathrm{SpO} 2)$ reported significantly lower in the death cohort than in survivors hospitalized in the intensive care unit (all $P<0.05$ ) (Table 2).

Further, both the groups were compared for biochemical profile and inflammatory markers. The findings showed that serum potassium level was significantly higher in patients with abnormal X-ray findings $(P<$
Table 2

Comparison of clinical characteristics among patients with COVID-19 $(\mathrm{N}=249)$.

\begin{tabular}{|c|c|c|c|}
\hline \multirow[t]{2}{*}{ Clinical characteristics } & \multicolumn{2}{|l|}{ Mean SD } & \multirow[t]{2}{*}{ p-value } \\
\hline & $\begin{array}{l}\text { Survivors ( } \mathrm{n}= \\
\text { 191) }\end{array}$ & $\begin{array}{l}\text { Non-Survivors }(\mathrm{n}= \\
58 \text { ) }\end{array}$ & \\
\hline $\begin{array}{l}\text { White blood cell count, } \times \\
10^{9} / \mathrm{L}\end{array}$ & $7.6 \pm 3.7$ & $8.7 \pm 6.0$ & $0.003^{*}$ \\
\hline Neutrophils count, $\times 10^{9} / \mathrm{L}$ & $6.5 \pm 1.5$ & $8.1 \pm 1.3$ & $<0.001$ \\
\hline $\begin{array}{l}\text { Lymphocytes count, } \times 10^{9} \text { / } \\
\text { L }\end{array}$ & $2.4 . \pm 1.4$ & $1.5 \pm 1.7$ & $<0.001$ \\
\hline Platelet, $\times 10^{9} / \mathrm{L}$ & $197.07 \pm 84.53$ & $171.58 \pm 113.40$ & 0.069 \\
\hline $\mathrm{Hb}, \mathrm{g} / \mathrm{L}$ & $12.66 \pm 2.19$ & $11.49 \pm 2.59$ & $0.001 *$ \\
\hline PT, s & $13.43 \pm 4.65$ & $18.10 \pm 9.67$ & $<0.001$ \\
\hline aPTT, s & $30.86 \pm 4.87$ & $51.10 \pm 2687$ & $<0.001$ \\
\hline Serum sodium, mmol/L & $138.24 \pm 4.46$ & $139.35 \pm 8.31$ & 0.191 \\
\hline Serum potassium, mmol/L & $4.18 \pm 0.60$ & $4.49 \pm 1.04$ & $0.005^{*}$ \\
\hline Serum chloride, mmol/L & $96.12 \pm 10.78$ & $94.65 \pm 8.75$ & 0.357 \\
\hline AST, U/L & $60.61 \pm 59.18$ & $88.16 \pm 124.84$ & $<0.001$ \\
\hline ALT, U/L & $49.94 \pm 58.12$ & $112.14 \pm 267.63$ & $0.003^{*}$ \\
\hline $\mathrm{LDH}, \mathrm{U} / \mathrm{L}$ & $635.18 \pm 298.09$ & $1356.00 \pm 1496.80$ & $0.007 *$ \\
\hline Direct bilirubin, mmol/L & $0.62 \pm 2.24$ & $0.73 \pm 1.50$ & 0.725 \\
\hline Indirect bilirubin, mmol/L & $0.49 \pm 1.54$ & $0.70 \pm 1.75$ & 0.395 \\
\hline TBIL, $\mathrm{mmol} / \mathrm{L}$ & $1.12 \pm 3.75$ & $1.43 \pm 3.21$ & 0.565 \\
\hline $\mathrm{CRP}, \mathrm{mg} / \mathrm{L}$ & $33.89 \pm 63.72$ & $161.25 \pm 116.26$ & $<0.001$ \\
\hline D-dimer, ng/mL & $709.00 \pm 739.01$ & $824.43 \pm 386.12$ & 0.689 \\
\hline CPK-MB & $36.24 \pm 36.48$ & $49.42 \pm 49.87$ & 0.229 \\
\hline Procal, ng/mL & $2.04 \pm 7.68$ & $24.25 \pm 22.69$ & $<0.001$ \\
\hline $\mathrm{PaO} 2, \mathrm{mmHg}$ & $90.83 \pm 11.87$ & $79.77 \pm 14.49$ & $<0.001$ \\
\hline $\mathrm{SpO} 2, \%$ & $95.98 \pm 4.66$ & $89.21 \pm 9.08$ & $<0.001$ \\
\hline
\end{tabular}

Table 3

Clinical characteristics based on radiological findings in patients with COVID-19 ( $\mathrm{N}=249$ ).

\begin{tabular}{llll}
\hline Clinical characteristics & \multicolumn{2}{l}{ X-Ray findings, Mean \pm SD } & \multirow{2}{*}{ p-value } \\
\cline { 2 - 3 } & Normal $(\mathrm{n}=$ & Abnormal $(\mathrm{n}=$ & \\
& $191)$ & $58)$ & \\
\hline White blood cell count, $\times 10^{9} /$ & $7.48 \pm 3.30$ & $7.96 \pm 5.65$ & $0.002^{*}$ \\
$\quad$ L & & & \\
Neutrophils count, $\times 10^{9} / \mathrm{L}$ & $62.49 \pm 13.91$ & $80.19 \pm 13.74$ & $<0.001$ \\
Lymphocytes count, $\times 10^{9} / \mathrm{L}$ & $29.89 \pm 12.73$ & $16.06 \pm 17.43$ & $<0.001$ \\
Platelet, $\times 10^{9} / \mathrm{L}$ & $200.66 \pm 90.81$ & $175.71 \pm 92.98$ & 0.038 \\
Hb, g/L & $12.58 \pm 2.43$ & $12.09 \pm 2.14$ & 0.113 \\
PT, s & $13.51 \pm 5.42$ & $16.08 \pm 7.53$ & $0.002^{*}$ \\
aPTT, s & $32.57 \pm 14.70$ & $39.44 \pm 10.49$ & 0.032 \\
Serum sodium, mmol/L & $138.13 \pm 4.40$ & $139.10 \pm 7.20$ & 0.184 \\
Serum potassium, mmol/L & $4.12 \pm 0.55$ & $4.46 \pm 0.93$ & $<0.001$ \\
Serum chloride, mmol/L & $96.17 \pm 11.51$ & $95.11 \pm 8.12$ & 0.437 \\
AST, U/L & $43.05 \pm 45.43$ & $86.12 \pm 112.77$ & $<0.001$ \\
ALT, U/L & $42.41 \pm 45.25$ & $100.41 \pm 215.81$ & $<0.001$ \\
LDH, U/L & $681.58 \pm 684.23$ & $827.62 \pm 471.90$ & 0.461 \\
Direct bilirubin, mmol/L & $0.69 \pm 2.49$ & $0.57 \pm 1.19$ & 0.629 \\
Indirect bilirubin, mmol/L & $0.54 \pm 1.70$ & $0.55 \pm 1.38$ & 0.957 \\
TBIL, mmol/L & $1.23 \pm 4.15$ & $1.12 \pm 2.54$ & 0.829 \\
CRP, mg/L & $20.88 \pm 46.17$ & $118.96 \pm 11.43$ & $<0.001$ \\
D-dimer, ng/mL & $713.63 \pm 774.60$ & $741.30 \pm 572.83$ & 0.890 \\
CPK-MB & $33.06 \pm 22.26$ & $49.64 \pm 59.02$ & 0.065 \\
Procal, ng/mL & $0.55 \pm 1.54$ & $16.73 \pm 21.38$ & $<0.001$ \\
PaO2, mmHg & $93.20 \pm 8.60$ & $79.94 \pm 18.69$ & $<0.001$ \\
SpO2, \% & $96.92 \pm 3.96$ & $90.19 \pm 7.93$ & $<0.001$ \\
\hline LDL & & & \\
& & &
\end{tabular}

LDL low-density lipoprotein; HDL high-density lipoprotein; TBIL total bilirubin; ALT alanine aminotransferase; AST aspartate transaminase; CK-MB creatinine kinase muscle and brain; APTT activated partial thromboplastin time; PT prothrombin time; CRP C -reactive protein; Proca procalcitonin; Hb hemoglobin; P $<0.05$ was considered significant.

0.05). However, there was no significant difference in both cohorts' chloride and sodium level (all $P>0.05$ ). There was no significant difference in liver studies observed on direct bilirubin, indirect bilirubin, total bilirubin, and lactate dehydrogenase status between both groups (all $P>0.05$ ). However, the level of alanine transaminase and aspartate transaminase were significantly higher among patients who reported 
abnormal x-ray findings (all $P<0.05$ )

There is a significant difference in C-reactive protein and procalcitonin levels between patients who reported abnormal $\mathrm{x}$-ray findings to counterparts (all $\mathrm{P}<0.05$ ) in terms of inflammatory markers. There was no significant difference in the lactate dehydrogenase and D-dimer levels between the cohort (all $P>0.05$ ). The oxygen saturation level and partial oxygen pressure were significantly lower in patients who reported abnormal on $x$-ray findings (all $P<0.05$ ) (Table 3 ).

\section{Discussion}

With the continuous spread of COVID-19 cases worldwide and different speculations of its effect on the human body are also flashing every day, we are still inexperienced in understanding a few aspects of COVID-19. However, we still have a lot to know about the effect of COVID-9 on different biochemical and hematological profiles in patients who survived or died due to COVID-19. Therefore, we summarised a comparative analysis of hematological and biochemical characteristics of 249 patients, analyzed the difference between survivors and death, and presented with abnormal $\mathrm{x}$-ray findings. This comparison is useful in the clinical setting to support clinical decisions and improving the survival rate in severely ill patients.

This study showed that the median age of the patients who died with COVID-19 was higher than the survived cohort. These findings are in agreement with the earlier research on patients with COVID-19. ${ }^{2,19}$ The older population have more number of comorbidities, limited organ function, reduced lung capacity, impaired immune system, biological aging, and more severe complications, these are the common reason pointed in earlier research on elderly with COVID-19, ${ }^{20,21}$ hence clinicians should treat them with more attention considering high-risk group.

Comparison of hematological findings showed that the death cohort had higher neutrophils count, white blood cells, higher prothrombin time, and activated partial thromboplastin time. Besides, the death cohort had lower lymphocyte count, platelets, and lower hemoglobin than survivors' cases. This conclusion mostly matches earlier studies conducted on patients with COVID-19. ${ }^{2,18,22}$ However, the role of platelet count in the mortality cohort should be described concerning the development of sepsis complications. Earlier work on sepsis patients in the intensive care unit discussed the role of platelet count in prognosis. ${ }^{2,23}$ However, thrombocytopenia's exact role is still not clear in terms of cause or consequence of patients with sepsis and need more research in this direction to reach a specific conclusion. Therefore, clinicians should keep a critical observation on patients with COVID-19 maintaining a track of low platelet count to act appropriately, including anti-inflammatory therapy or platelet transfusion if required. In severe cases, developing thrombocytopenia can mean more severe damage leads to more substantial damage to the immune system. ${ }^{2}$

There were also significant differences among alanine transaminase, aspartate transaminase, C-reactive protein, procalcitonin, and lactate dehydrogenase between survivors and death cohorts, which are similar to earlier research on COVID-19. 2,9,24 Earlier studies said that level of procalcitonin remains normal in patients with viral infections. ${ }^{25}$ In our findings, the procalcitonin level was significantly higher in the death cohort than that of the survived group indicate the possibility of multiple infections at a time. Besides, we found a low level of serum potassium in COVID-19 cases. These findings agree with a study that reported a negative correlation of potassium with the disease severity in COVID-19 cases. ${ }^{9}$ This is because a higher level of ACE 2 degradation impairs renal control on potassium and makes it challenging for the clinicians to manage hypokalaemia in a patient with SARS-COV-2.

In the present study, many other biochemical indicators showed a significant difference between survivors and death cohort, such as AST,
ALT, and LDH. The abnormalities in these indicators suggested that SARS-COV-2 infection may harm hepatic, myocardial, and other human body organs. These findings are an exact match to the earlier research on patients with COVID-19 disease. ${ }^{2,9}$ However, some clinical indicators reported controversy in the present work compared to earlier work, including-D-dimer and platelet count in patients with COVID-19. The change in findings may be due high mortality among patients with COVID-19 in initial days and a lack of complete information on the status of D-dimer in all patients with COVID-19. Controversial presentation of clinical characteristics among patients with COVID-19 need further research on large sample across multiple centres to reach on a specific conclusion.

As per this study finding concern, patient characteristics, including age, leucocyte count, platelet count, inflammatory markers status, hypokalaemia, and blood oxygen saturation, may be significant predictors for developing critical illness SARS-COV-2 patients. However, these parameters need further evaluation in a larger number of cases and should be used only for risk stratification purposes. COVID-19 is an unpredictable, viral attack and may take a rapid turn to develop a severe illness. Therefore, high-risk patients need strict monitoring round the clock and supportive management timely.

\subsection{Limitations}

The study should be seen under many limitations. First, a retrospective cross-sectional study is limited to a single center and provides no information on a cause to effect relationship. Secondly, only a limited number of hematological and biochemical markers are studied, limiting the findings' implications. These markers and clinical characteristics need further investigations and should be used for risk stratification in patients with COVID-19. This study provides new insight into the substantial role of other clinical characteristics in patients with COVID-19. By studying a large number of cases and the autopsy information, we will surely get more precise information on clinical characteristics and their role in treating the disease. This will further help to formulate a robust plan to control and mitigate the pandemic at large.

\section{Authors' contributions}

Conceptualization: MB, RK, KB. Data curation: MB, RK. Formal analysis: YB. Methodology: MB, RK, KB. Project administration: MB, KB. Visualization: MR, RK. Writing - RK, DK: Writing - review \& editing: DK, $\mathrm{KB}$

\section{Financial disclosures}

None.

\section{Ethical permission}

Obtained (AIIMS/IEC/20/442).

LDL low-density lipoprotein; HDL high-density lipoprotein; TBIL total bilirubin; ALT alanine aminotransferase; AST aspartate transaminase; CK-MB creatinine kinase muscle and brain; APTT activated partial thromboplastin time; PT prothrombin time; CRP C -reactive protein; Proca procalcitonin; $\mathrm{Hb}$ hemoglobin; $\mathrm{P}<0.05$ was considered significant.

\section{Declaration of competing interest}

No potential conflict of interest relevant to this article was reported. 


\section{Appendices.}

In the cohort, $26.5 \%$ of patients advanced symptoms of critical illness. The rest were considered non-critical as per Indian treatment guidelines issued by the Ministry of Health and Family Welfare (MoHFW), Directorate General of Health Services (DGHS). The summary of the findings is presented in supplementary file 1.

\section{Supplementary File}

Demographic and clinical characteristics of SARS-COV-2 patients $(\mathrm{N}=249)$.

\begin{tabular}{|c|c|}
\hline Characteristics & Total, Mean (SD), [Range] \\
\hline No & 249 \\
\hline Age (mean SD) years & 45.15 (16.35) [7-85] \\
\hline$<40$ & $118(47.39)$ \\
\hline$\geq 40$ & $117(46.99)$ \\
\hline \multicolumn{2}{|l|}{ Gender } \\
\hline Male & $171(68.67)$ \\
\hline Female & $78(31.33)$ \\
\hline \multicolumn{2}{|l|}{ Admission criteria } \\
\hline \multicolumn{2}{|l|}{ Temperature } \\
\hline Febrile & $112(44.98)$ \\
\hline Afebrile & $137(55.02)$ \\
\hline Respiratory rate, (/mints) & $21.81(4.01)[14-38]$ \\
\hline Heart rate, $\mathrm{b} / \mathrm{mints}$ & $97.76(19.82)[50--169]$ \\
\hline \multicolumn{2}{|l|}{ Blood pressure (mmHg) } \\
\hline Systolic (SBP) & $126.9(21.24)[60--260]$ \\
\hline Diastolic (DBP) & $78.68 /(14.49)[40--140]$ \\
\hline \multicolumn{2}{|l|}{ Smoking status } \\
\hline Former/Current & $47 / 249(18.88)$ \\
\hline History of travel & $66 / 249(26.51)$ \\
\hline Comorbidity^ & $86 / 249(34.54)$ \\
\hline CKD & $12 / 249(4.82)$ \\
\hline CAD & $18 / 249(7.23)$ \\
\hline HTN & $49 / 249(19.68)$ \\
\hline $\mathrm{DM}$ & $49 / 249(19.68)$ \\
\hline COPD & $31 / 249(12.45)$ \\
\hline Immunodeficiency & $14 / 249(5.62)$ \\
\hline \multicolumn{2}{|l|}{ Signs-symptoms } \\
\hline Fever & $96 / 249(38.55)$ \\
\hline Cough & $62 / 249(24.9)$ \\
\hline Abnormal cardiac rhythm & $07 / 249(2.8)$ \\
\hline Dyspnea & $80 / 249(32.1)$ \\
\hline Sore throat & $18 / 249(7.2)$ \\
\hline Myalgia & $10 / 249(4.5)$ \\
\hline Headache & $07 / 249(2.8)$ \\
\hline Productive cough & $18 / 249(7.2)$ \\
\hline Nausea \& vomiting & $8 / 249(3.21)$ \\
\hline Diarrhea & $18 / 249(7.23)$ \\
\hline Unconsciousness & $66 / 249(26.51)$ \\
\hline
\end{tabular}

\section{References}

1 Li Q, Guan X, Wu P, et al. Early transmission dynamics in wuhan, China, of novel coronavirus-infected pneumonia. New England Journal of Medicine. 2020;382: 1199-1207. https://doi.org/10.1056/NEJMoa2001316. Available from:.

2 Sheng L, Wang X, Tang N, et al. Clinical characteristics of moderate and severe cases with COVID-19 in Wuhan, China: a retrospective study. Clinical and Experimental Medicine. 2020. https://doi.org/10.1007/s10238-020-00662-z. Available from:.

3 Kumar R, Singh V, Mohanty A, Bahurupi Y, Gupta PK. Corona health- care warriors in India: knowledge, attitude, and practices during COVID-19 outbreak. J Educ Health Promot. 2021;10(44):1-8. https://doi.org/10.4103/jehp.jehp_524_20.

4 Wang D, Hu B, Hu C, et al. Clinical characteristics of 138 hospitalized patients with 2019 novel coronavirus-infected pneumonia in wuhan, China. JAMA. 2020;323: 1061-1069. https://doi.org/10.1001/jama.2020.1585. Available from:.

5 Gautier J-F, Ravussin Y. A new symptom of COVID-19: loss of taste and smell. Obesity. 2020:848.

6 World Health Organization World Health Organization. Statement on the second meeting of the international health regulations (2005). Emergency Committee regarding the outbreak of novel coronavirus (2019-nCoV); 2020. Available from: https://www.who.int/news-room/detail/30-01-2020-statement-on-th e-second-meeting-of-the-international-health -regulations-(2005)-emergency-committee-regarding-th e-outbreak-of-novel-coronavirus-(2019-ncov. Accessed January 13, 2021.
7 Lu J, Hu S, Fan R, et al. ACP risk grade: a simple mortality index for patients with confirmed or suspected severe acute respiratory syndrome coronavirus 2 disease (COVID-19) during the early stage of outbreak in Wuhan. medRxiv China; 2020. p. 2020.02.20.20025510. Available from: http://medrxiv.org/content/ early/2020/02/23/2020.02.20.20025510.abstract. Accessed January 13, 2021.

8 Liu J, Liu Y, Xiang P, et al. Neutrophil-to-Lymphocyte ratio predicts severe illness patients with 2019. medRxiv Novel Coronavirus in the Early Stage; 2020. p. 2020.02.10.20021584. Available from: http://medrxiv.org/content/early/2020/ 02/12/2020.02.10.20021584.abstract. Accessed January 13, 2021.

9 Wang D, Li R, Wang J, et al. Correlation analysis between disease severity and clinical and biochemical characteristics of 143 cases of COVID-19 in Wuhan, China: a descriptive study. BMC Infectious Diseases. 2020;20:519. https://doi.org/10.1186/ s12879-020-05242-w. Available from:.

10 Tian S, Liu H, Liao M, et al. Analysis of mortality in patients with COVID-19: clinical and laboratory parameters. Open Forum Infectious Diseases. 2020:7. https://doi.org/ 10.1093/ofid/ofaa152. Available from:.

11 Wang K, Qiu Z, Liu J, et al. Analysis of the clinical characteristics of 77 COVID-19 deaths. Scientific Reports. 2020;10:16384. https://doi.org/10.1038/s41598-02073136-7. Available from:.

12 Martins-Filho PR, Tavares CSS, Santos VS. Factors associated with mortality in patients with COVID-19. A quantitative evidence synthesis of clinical and laboratory data. European journal of internal medicine. 2020;76:97-99. Available from: https://pubmed.ncbi.nlm.nih.gov/32345526. Accessed April 23, 2020. 
13 Emery SL, Erdman DD, Bowen MD, et al. Real-time reverse transcription-polymerase chain reaction assay for SARS-associated coronavirus. Emerging infectious diseases. 2004;10:311-316. Available from: https://pubmed.ncbi.nlm.nih.gov/15030703.

14 Indian Council of Medical Research. National Institute of epidemiology. COVID-19 sample collection guidelines. Available from: http://www.nie.gov.in/images/leftcon tent_attach/COVID-SARI_Sample_collection_SOP_255.pdf; 2020. Accessed January $18,2021$.

15 ThermoFisher Scientific. TaqPathTM COVID-19 combo kit. Available from: https://www.thermofisher.com/order/catalog/product/A47814\#/A47814; 2020. Accessed January 18, 2021.

16 Government of India. Ministry of health \& family Welfare Services DG of HD (EMR). Revised guidelines on clinical management of COVID - 19. Available from: https ://www.mohfw.gov.in/pdf/RevisedNationalClinicalManagementGuidelinefor COVID1931032020.pdf; 2020. Accessed January 16, 2021.

17 Metlay JP, Waterer GW, Long AC, et al. Diagnosis and treatment of adults with community-acquired pneumonia. An official clinical practice guideline of the American thoracic society and infectious diseases society of America. American Journal of Respiratory and Critical Care Medicine. 2019;200:e45-e67. https://doi.org/ 10.1164/rccm.201908-1581ST. Available from:

18 Liang W, Liang H, Ou L, et al. Development and validation of a clinical risk score to predict the occurrence of critical illness in hospitalized patients with COVID-19. JAMA internal medicine. 2020;180:1081-1089. https://pubmed.ncbi.nlm.nih.gov /32396163.
19 Qin C, Zhou L, Hu Z, et al. Dysregulation of immune response in patients with coronavirus 2019 (COVID-19) in wuhan, China. Clin Infect Dis: an official publication of the Infectious Diseases Society of America. 2020;71:762-768.

20 Shahid Z, Kalayanamitra R, McClafferty B, et al. COVID-19 and older adults: what we know. Journal of the American Geriatrics Society. 2020;68:926-929. Available from https://pubmed.ncbi.nlm.nih.gov/32255507. Accessed April 20, 2020.

21 Kang SJ, Jung SI. Age-related morbidity and mortality among patients with COVID19. Infection \& chemotherapy. 2020;52:154-164, 2020/06/12, Available from: https://pubmed.ncbi.nlm.nih.gov/32537961.

22 Shang Y, Liu T, Wei Y, et al. Scoring systems for predicting mortality for severe patients with COVID-19. EClinicalMedicine. 2020;24:100426. Available from: http:// www.sciencedirect.com/science/article/pii/S258953702030170X.

23 Assinger A, Schrottmaier WC, Salzmann M, et al. Platelets in sepsis: an update on experimental models and clinical data. Front Immunol. 2019;10:1687.

24 Liu J, Li S, Liu J, et al. Longitudinal characteristics of lymphocyte responses and cytokine profiles in the peripheral blood of SARS-CoV-2 infected patients. EBioMedicine. 2020;55:102763. Available from: http://www.sciencedirect.com/sci ence/article/pii/S2352396420301389.

25 Albrich WC, Harbarth S. Pros and cons of using biomarkers versus clinical decisions in start and stop decisions for antibiotics in the critical care setting. Intensive Care Med. 2015;41:1739-1751. 